\title{
Universal enthalpy-entropy compensation rule for the deformation of metallic glasses
}

\author{
Yun-Jiang Wang, ${ }^{1, *}$ Meng Zhang,,${ }^{1,2}$ Lin Liu, ${ }^{2}$ Shigenobu Ogata,,${ }^{3,4, \dagger}$ and L. H. Dai ${ }^{1, \ddagger}$ \\ ${ }^{1}$ State Key Laboratory of Nonlinear Mechanics, Institute of Mechanics, Chinese Academy of Sciences, Beijing 100190, China \\ ${ }^{2}$ School of Materials Science and Engineering, Huazhong University of Science and Technology, Wuhan 430074, China \\ ${ }^{3}$ Graduate School of Engineering Science, Osaka University, Osaka 560-8531, Japan \\ ${ }^{4}$ Center for Elements Strategy Initiative for Structural Materials (ESISM), Kyoto University, Sakyo, Kyoto 606-8501, Japan \\ (Received 3 July 2014; revised manuscript received 14 October 2015; published 23 November 2015; corrected 1 December 2015)
}

\begin{abstract}
The thermodynamic compensation law describing an empirical linear relationship between activation enthalpy and activation entropy has seldom been validated for amorphous solids. Here molecular dynamics simulations reveal a well-defined enthalpy-entropy compensation rule in a metallic glass (MG) over a wide temperature and stress range, spanning the glass transition induced by temperature and/or stress. Experiments on other MGs reproduce this law, suggesting that it applies universally to amorphous solids, so we extend it from crystals to amorphous solids. In the glassy state, the compensation temperature is found to agree with the thermal glass transition temperature $T_{\mathrm{g}}$; whereas in the supercooled liquid region, the compensation temperature matches $\sim 1.4 T_{\mathrm{g}}$, at which the diffusion kinetics start to feel the roughness of the free-energy surface.
\end{abstract}

DOI: 10.1103/PhysRevB.92.174118

\section{INTRODUCTION}

The ubiquitous thermodynamic enthalpy-entropy compensation rule was initially discovered in chemistry $[1,2]$ and biology [3], and was then extended to condensed-matter physics [4] and materials science [5-8]. It indicates a linear relationship between the activation entropy and the enthalpy [9-11]. Despite decades of studies of this effect in crystals, it has not been validated in amorphous solids. In this paper, combined atomistic simulations and experiments reveal a universally well-established enthalpy-entropy compensation in the deformation of metallic glasses (MGs), although the structure and deformation units of these two phases differ sharply from each other [12-14].

Transition state theory (TST) is a general statistical law explaining the rates of chemical reactions [15]. It is nowadays prevalent to describe the plastic deformation of material, which is usually driven by thermally activated processes $[16,17]$. TST predicts the occurrence frequency of a plastic event using the Arrhenius equation, which associates the activation Gibbs free energy $\Delta G$ with the resultant rate $\nu$, i.e.,

$$
\nu=v_{0} \exp \left(-\frac{\Delta G(\tau, T)}{k_{B} T}\right) .
$$

For shear-stress-driven deformation processes, the rate is a function of the shear stress $\tau$ and temperature $T . v_{0}$ is an attempt frequency. Further, $k_{B}$ is Boltzmann's constant and $k_{B} T$ is the thermal energy. Finally, $\Delta G$ is

$$
\Delta G(\tau, T)=\Delta Q-\tau \Omega-T \Delta S .
$$

Here, $\Delta Q$ is the activation energy and $\Omega$ is the activation volume. $\Delta H=\Delta Q-\tau \Omega$ denotes activation enthalpy and $\Delta S$ is the activation entropy. The above theoretical framework constitutes the fundamentals of material deformation physics.

The thermodynamic compensation rule, or Meyer-Neldel (MN) rule [9], indicates an empirical linear relationship

\footnotetext{
*yjwang@imech.ac.cn

${ }_{\dagger}^{\dagger}$ ogata@me.es.osaka-u.ac.jp

${ }^{\ddagger}$ Ihdai@lnm.imech.ac.cn
}

PACS number(s): 61.43.Dq, 05.70.-a, 61.20.Ja, 82.20.Db

between the activation entropy and activation enthalpy,

$$
\Delta S=\frac{\Delta H}{T_{\mathrm{MN}}},
$$

where $T_{\mathrm{MN}}$ is a characteristic temperature called the $\mathrm{MN}$ temperature [10,11]. MN rule predicts $\Delta G=\left(1-T / T_{\mathrm{MN}}\right) \Delta H$. It has been found to be ubiquitous in a vast number of chemical phenomena [18], condensed-matter physics [4], and biological processes [3]. Recently, it has been more frequently reported in the plastic deformation mechanisms of crystals, e.g., diffusion [8], interface and grain boundary mobilities [5], and dislocation behaviors $[6,7]$.

Although the $\mathrm{MN}$ rule seems to apply universally to crystals, it is interesting to know whether it stands or break down in amorphous solids [19]. To date, a compensation effect has been reported for diffusion in MGs between the diffusion prefactor and activation enthalpy [20]. In contrast with crystals, the deformation unit of MGs has not been completely recognized [21]. At low temperature, the plastic event is commonly assumed to be a collective local shear of atoms. The scenarios include the shear transformation zone (STZ) [22,23], cooperative shear model (CSM) [24,25], flow unit [26-29], and tensile transformation zone (TTZ) [30,31]. The STZ represents a thermally activated displacive process [32,33] with a typical activation energy of multiple tens of $k_{B} T$ and an activation volume of dozens of atoms [34-36]. Further, at high temperature, a diffuse arrangement of atoms is also involved. In this work, we confirm the validation of the enthalpy-entropy compensation effect in MGs over a wide temperature and stress range, and discuss the possible correlation of the compensation effect with important critical temperatures in MGs.

\section{METHODOLOGY}

\section{A. Atomistic simulations}

The present MD simulation is performed using the LAMMPS code [37]. An embedded-atom method (EAM) potential, which has been extensively verified by both first-principles 
(a)
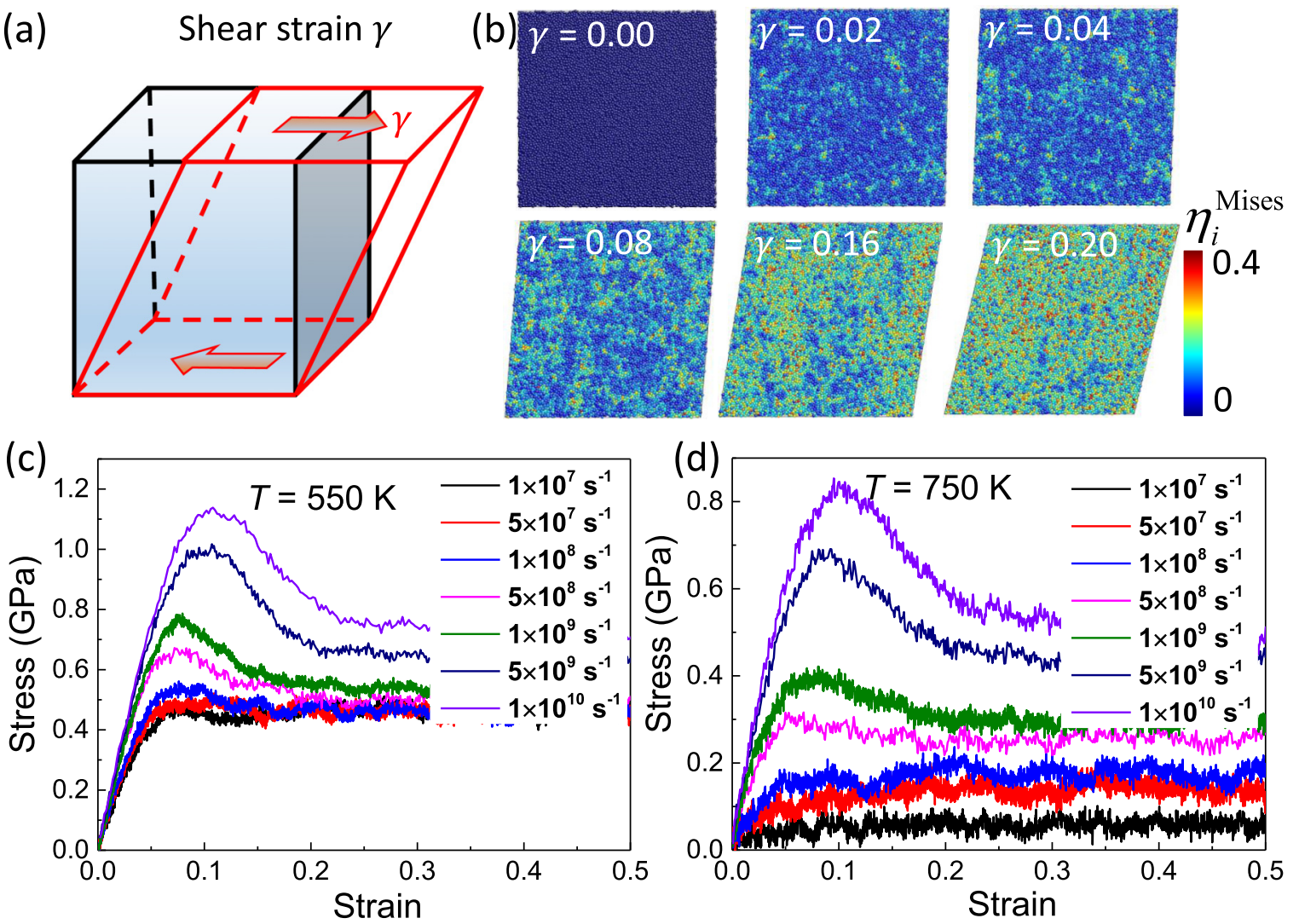

FIG. 1. (Color online) Atomistic shear deformation of $\mathrm{Cu}_{50} \mathrm{Zr}_{50}$. (a) Schematic sketch of the atomistic shear deformation. (b) MD snapshots indicating homogeneous shear deformation with strain rate $10^{8} \mathrm{~s}^{-1}$ and temperature $600 \mathrm{~K}$. Atoms are colored according to their local von Mises strain $\eta_{i}^{\text {Mises }}$ of atom $i$ [47], which is implemented in ATOMEYE [48]. Typical stress-strain curves at (c) 550 and (d) $750 \mathrm{~K}$, respectively.

calculations and experiments [38], is adopted to describe the interatomic interaction. This potential has been widely used to investigate the structure and mechanics of many multicomponent MGs [39-41]. Here a $\mathrm{Cu}_{50} \mathrm{Zr}_{50}$ glass model is prepared by a melting-quenching technique with a cooling rate of $10^{12} \mathrm{~K} / \mathrm{s}$. The model contains 100000 atoms, with dimensions of $20 \times 20 \times 4 \mathrm{~nm}^{3}$ for the $X, Y$, and $Z$ directions, respectively. The glass transition temperature of $\mathrm{Cu}_{50} \mathrm{Zr}_{50}$ is estimated as $T_{\mathrm{g}}=675 \mathrm{~K}$ by $\mathrm{MD}$, which is in agreement with experimental data $[42,43]$. Note that in MGs, shear is the dominant deformation mode, even though compression or tension is more frequently applied in experiments $[22,34,44]$. Therefore, we conduct straightforward shear deformation in these computer simulations. Shear deformation is applied with a constant strain rate between $10^{7}$ and $10^{10} \mathrm{~s}^{-1}$ for the engineering shear strain $\gamma_{x y}$; see Fig. 1(a). The constant strain rate is applied by incrementally shearing the simulation box at each MD step. The MD time step is set to be $2 \mathrm{fs}$. All of the other stress components except $\tau_{x y}\left(=\tau_{y x}\right)$ are kept at 0 during deformation. Simulations are conducted within an isothermalisobaric ensemble via a Parrinello-Rahman technique [45]. All of the samples are thermally equilibrated sufficiently for $200 \mathrm{ps}$ before shearing. We choose the temperature range of [500 K, $850 \mathrm{~K}$ ], which is around the $T_{\mathrm{g}}$ value of $\mathrm{Cu}_{50} \mathrm{Zr}_{50}$. The above conditions guarantee that the deformation lies on the homogeneous flow regime of the MG deformation map [34], where thermal activation is believed to dominate the plastic deformation [46].

\section{B. Activation free energy}

In a steady-state flow, the effective shear rate $\dot{\gamma}$ usually obeys a general rate law $[22,34,49]$ :

$$
\dot{\gamma}=\dot{\gamma}_{0} \exp \left[-\frac{\Delta G(\tau, T)}{k_{B} T}\right] .
$$

For the STZ, $\dot{\gamma}_{0}=\alpha_{0} v_{0} \gamma_{0}$ is a shear rate prefactor that establishes the upper limit of the strain rate. $\alpha_{0}$ (of order unity) is a parameter incorporating the volume fraction of glass that is ready to deform and other numerical factors [22,34]. $v_{0} \sim$ $10^{11}-10^{13} \mathrm{~s}^{-1}$ is the attempt frequency, which depends on the diameter of the deformation unit [49]. $\gamma_{0} \sim 0.1$ is the unit strain at which STZs transform on average $[22,34,46]$. The above considerations yield a reasonable characteristic shear rate on the order of $10^{10}-10^{12} \mathrm{~s}^{-1}$, which is commonly assumed in the literature $[22,34,50]$. Our MD predicts $\dot{\gamma}_{0}=1.2 \times 10^{10} \mathrm{~s}^{-1}$ (see the details in Fig. S1 of the Supplemental Material [51]), which agrees with the above analysis [22,34]. If we rewrite Eq. (4) as

$$
\Delta G(\tau, T)=k_{B} T \ln \left(\dot{\gamma}_{0} / \dot{\gamma}\right),
$$

then $\Delta G$ can be derived as a function of both $T$ and $\tau$ on the basis of the plots in Fig. 2(a). Note that a change of even one order of magnitude in $\dot{\gamma}_{0}$ will cause variations of only $\sim 2 k_{B} T$ in the free energy and $\sim 2 k_{B}$ in the entropy; the uncertainty in the prefactor $\dot{\gamma}_{0}$ does not substantially change the main conclusions based on the free energy, which is multiple tens 

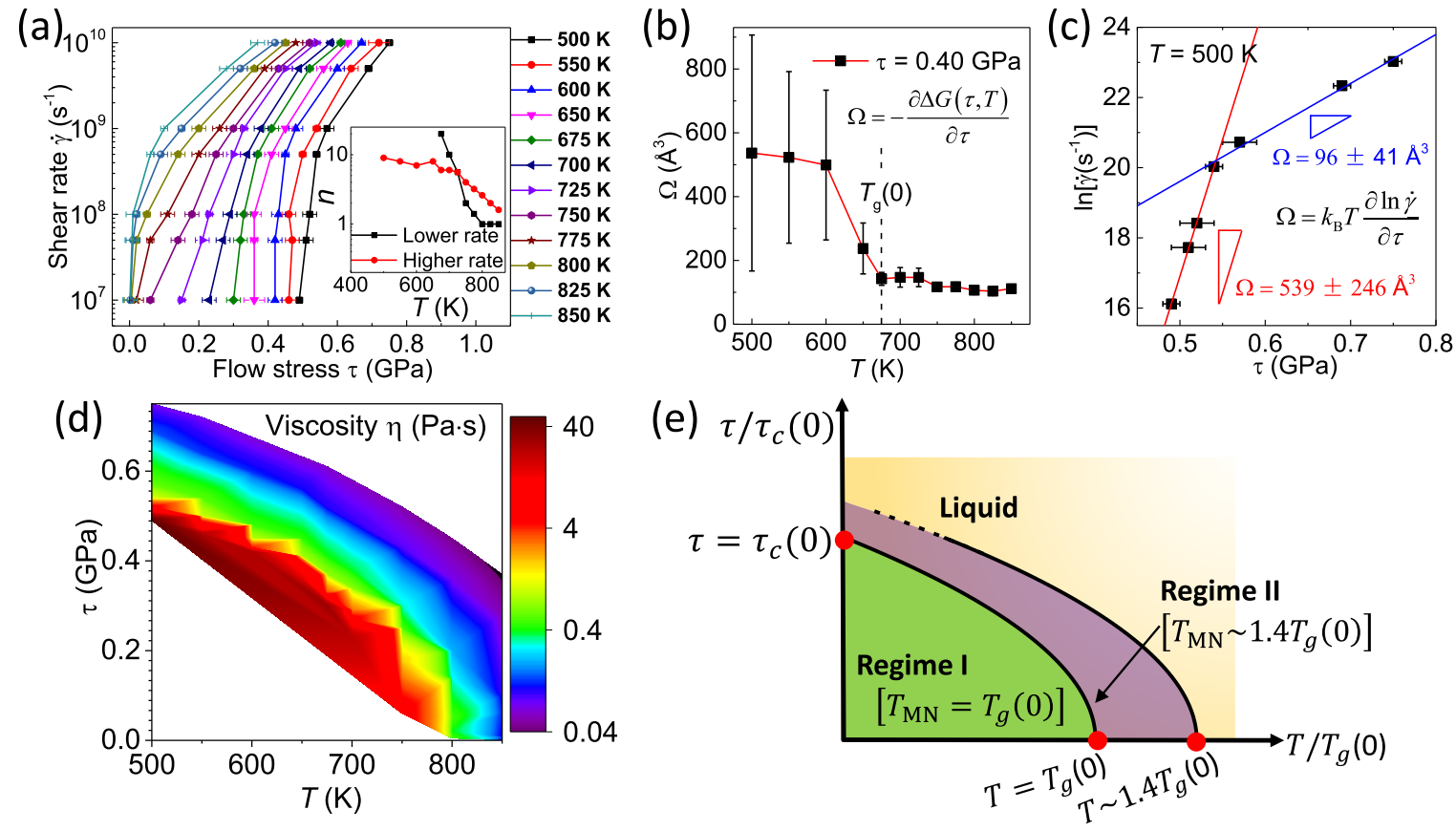

FIG. 2. (Color online) Temperature- and stress-driven transition of deformation mechanisms in MG. (a) Correlation between strain rate and flow stress. Inset shows the variation in the stress exponent $n$. (b) Activation volume decreases abruptly at temperature well below $T_{\mathrm{g}}(0)$, denoting a temperature- and stress-induced glass transition and a shift in the deformation mechanism. (c) Logarithmic strain rate vs stress. Slope change indicates a decrease in activation volume, which implies a stress-induced glass transition. (d) Viscosity contour against both temperature and stress. Plots indicate the glassy state in a low-temperature and low-stress regime, whereas the glass transition can be driven by either temperature or stress. (e) Schematics of deformation regimes defined by $T_{\mathrm{g}}(0)$ and the temperature-dependent critical stress $\tau_{\mathrm{c}}(T)$. Regime I (glassy state): $T<T_{\mathrm{g}}(0), \tau<\tau_{\mathrm{c}}(T)$, where $\tau_{\mathrm{c}}(T)$ is determined by $\frac{T}{T_{\mathrm{g}}(0)}+\left[\frac{\tau}{\tau_{\mathrm{c}}(0)}\right]^{2}=1$. Regime II (supercooled liquid): $\tau>\tau_{\mathrm{c}}(T)$, but within $\frac{T}{T_{\mathrm{g}}(0)}+\left[\frac{\tau}{\tau_{\mathrm{c}}(0)}\right]^{2}=1.4$. Regime III (liquid): outside regime II.

of $k_{B} T$. By employing Eq. (4), we have taken the statistics of competing processes with scattered energy barriers as a single activation process in MG deformation, which has been theoretically verified previously by an activation-relaxation technique in MD [19].

\section{RESULTS}

\section{A. Temperature- and stress-driven transition of deformation mechanisms}

We simulate $\mathrm{Cu}_{50} \mathrm{Zr}_{50} \mathrm{MG}$ shear deformation over a wide temperature range $(500-850 \mathrm{~K})$ that extends both below and above $T_{\mathrm{g}}=675 \mathrm{~K}$, which is the stress-dependent glass transition temperature [39] at the stress free condition: $\left.T_{\mathrm{g}}(\tau)\right|_{\tau=0}=T_{\mathrm{g}}(0)=T_{\mathrm{g}}$. This temperature range allows for the homogeneous shear of the glass model; see Figs. 1(a) and 1(b) for simulations [51]. Figures 1(c) and 1(d) show that the flow stress is more strain-rate sensitive at high temperature, which indicates that the deformation unit becomes less collective (activation volume is inversely proportional to the rate sensitivity).

The strain rate is displayed as a function of the flow stress at different temperatures in Fig. 2(a). The inset of Fig. 2(a) shows that Newtonian flow could be obtained only in the low-strainrate and high-temperature regime, which is marked by a stress exponent $n=1$ (if a power law of $\dot{\gamma} \propto \tau^{n}$ is assumed, and $n=\partial \ln \dot{\gamma} \partial \ln \tau$ ). However, the majority of the deformation shows a collective nature, indicated by a large stress exponent
( $n>1$, non-Newtonian flow). The inset of Fig. 2(a) also shows that the stress exponents are different at high- and low-strainrate regimes, such as $\dot{\gamma}>5 \times 10^{8} \mathrm{~s}^{-1}$ and $\dot{\gamma}<5 \times 10^{8} \mathrm{~s}^{-1}$. The threshold $\dot{\gamma} \sim 5 \times 10^{8} \mathrm{~s}^{-1}$ is empirically chosen from the kink of the rate-stress plot.

The transition of the stress exponent indicates the temperature- and stress-induced transition of deformation mechanisms. Figure 2(b) shows that the activation volume [ $\Omega=-\partial \Delta G / \partial \tau$, based on Figs. 3(a) and 3(b) below] is dramatically decreased by increasing the temperature above $T_{\mathrm{g}}(0)$; thus, the thermal glass transition actually introduces the transition in the deformation mechanism. The size of the activation volume here is similar to that estimated experimentally [35]. At high temperature, viscous flow involving a diffusive mechanism dominates the deformation. Figure 2(c) shows that stress can also induce the transition in the deformation mechanism. The activation volume at $500 \mathrm{~K}$ (derived as $\left.\Omega=k_{B} T \partial \ln \dot{\gamma} / \partial \tau\right)$ varies from about $500 \AA^{3}$ to about $100 \AA^{3}$ with increasing stress. The latter resembles the activation volume after the thermal glass transition, as shown in Fig. 2(b), which suggests that stress could also drive a glass transition even below $T_{\mathrm{g}}(0)$. This scenario of a stress-induced glass transition agrees with early speculation [52] and atomistic demonstration [39]. Figure S2 of the Supplemental Material provides further evidence for this point [51].

The viscosity contour $\eta(\tau, T)=\tau / \dot{\gamma}_{0} \exp \left(\Delta G / k_{B} T\right)$ shown in Fig. 2(d) intuitively characterizes the glass transition induced by temperature, stress, or both. Temperature and stress 

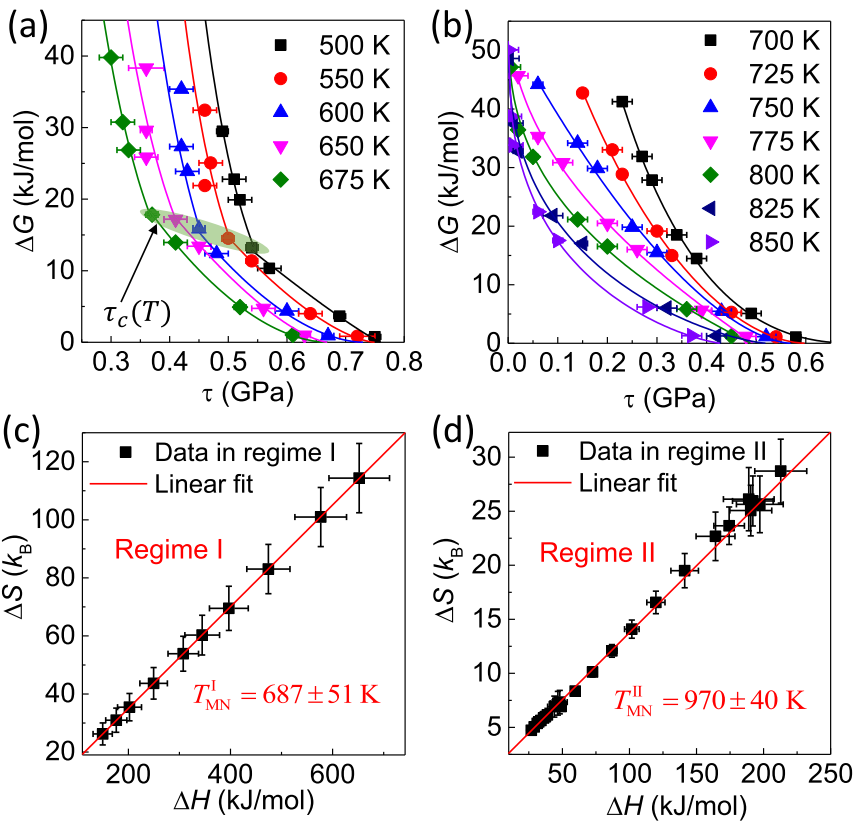

FIG. 3. (Color online) Enthalpy-entropy compensation rule in MG revealed by MD. (a), (b) Activation free energy as a function of stress at temperatures above and below $T_{\mathrm{g}}(0)$, respectively. Solid curves represent nonlinear fits. Shaded area in (a) denotes a stressinduced transition of the deformation mechanism. (c), (d) Established linear relationship between $\Delta S$ and $\Delta H$ in (c) regime I and (d) regime II.

play a similar role in decreasing the viscosity and consequently in the glass transition. The activation volume and viscosity consistently verify the existence of both temperature- and stress-induced glass transitions. Regarding the essential role of temperature- and stress-induced glass transitions, we tentatively divide the $T-\tau$ deformation map into three regimes with a stress-temperature scaling law proposed in Ref. [39]: (I) $T<T_{\mathrm{g}}(0), \tau<\tau_{\mathrm{c}}(T)$, where the temperature-dependent critical stress $\tau_{\mathrm{c}}(T)$ is determined by $\frac{T}{T_{\mathrm{g}}(0)}+\left[\frac{\tau}{\tau_{\mathrm{c}}(0)}\right]^{2}=1$, with $T_{\mathrm{g}}(0)=675 \mathrm{~K}$, and $\tau_{\mathrm{c}}(0)=1.1 \mathrm{GPa}$ the athermal stress estimated from MD; (II) $\tau>\tau_{\mathrm{c}}(T)$, but within $\frac{T}{T_{\mathrm{g}}(0)}+\left[\frac{\tau}{\tau_{\mathrm{c}}(0)}\right]^{2}=$ 1.4; and (III) outside regime II. Regimes I, II, and III represent glass, supercooled liquid, and liquid, respectively. The present MD data lie in regimes I and II. The choice of the empirical value $T \sim 1.4 T_{\mathrm{g}}$ will be discussed later.

\section{B. Enthalpy-entropy compensation discovered in MG through atomistic simulations}

Next, we demonstrate the enthalpy-entropy compensation for each regime. Figures 3(a) and 3(b) plot $\Delta G$ obtained from Eq. (5) against the stress at temperatures below and above $T_{\mathrm{g}}(0)$, respectively. A frequently used empirical powerlaw equation, $\Delta G=\Delta F\left\{1-\left[\tau / \tau_{\mathrm{c}}(0)\right]^{p}\right\}^{q}$, is employed to interpolate the free-energy data points $[24,49,53]$. Here, $\Delta F=$ $\Delta Q-T \Delta S$ is the activation Helmholtz free energy, $\tau_{\mathrm{c}}(0)$ is the athermal stress, and $0<p \leqslant 1$ and $q \geqslant 1$ are parameters. The shaded area in Fig. 3(a) implies a transition in mechanism induced by stress. For an accurate description, we use separate curves with different $p$ and $q$ to fit the data in Fig. 3(a) before and after $\tau_{\mathrm{c}}(T)$. On the other hand, Fig. 3(b) shows that $\Delta G$ versus $\tau$ could be described well by a single curve when $T>T_{\mathrm{g}}(0)$, indicating no change in the mechanism. As a result, the available $\Delta G(\tau, T)$ has been divided into two regimes, including the data before and after the glass transition, as proposed in Fig. 2(e), regardless of whether the transition is driven by temperature or stress. Then we extract $\Delta G$ as a function of $T$ for multiple stress levels; see Fig. S3 in Supplemental Material [51]. Linear fits based on Eq. (2) yield $\Delta S$ and $\Delta H$.

Finally, we can confirm the enthalpy-entropy correlation in MGs. Figures 3(c) and 3(d) present well-defined linear relationships between $\Delta S$ and $\Delta H$ for the two regimes. The characteristic temperatures $T_{\mathrm{MN}}$ are determined from linear fittings as (I) $T_{\mathrm{MN}}^{\mathrm{I}}=687 \pm 51 \mathrm{~K}$ and (II) $T_{\mathrm{MN}}^{\mathrm{II}}=970 \pm 40 \mathrm{~K}$, respectively. In the glassy state, $T_{\mathrm{MN}}^{\mathrm{I}}=687 \pm 51 \mathrm{~K}$ for the dominant mechanism below $\tau_{\mathrm{c}}(T)$. It is very close to $T_{\mathrm{g}}(0)=$ $675 \mathrm{~K}$. In the supercooled liquid regime, the compensation temperature is about $1.4 T_{\mathrm{g}}(0)$.

\section{Experimental support for enthalpy-entropy compensation rule in MGs}

The MD informed compensation rule is also supported by compressive experiments on Vitreloy 1 (Vit 1) by Lu et al. [54], as show in Fig. 4. Figure 4(a) shows how the flow stress is adjusted by the strain rate. Since compression is applied in experiments, we redefine $\Delta G=\Delta H-T \Delta S=$ $\Delta Q-\sigma \Omega^{\prime}-T \Delta S$, where $\sigma$ is the compressive stress and $\Omega^{\prime}$ is its conjugate activation volume. $\Delta G$ could be obtained
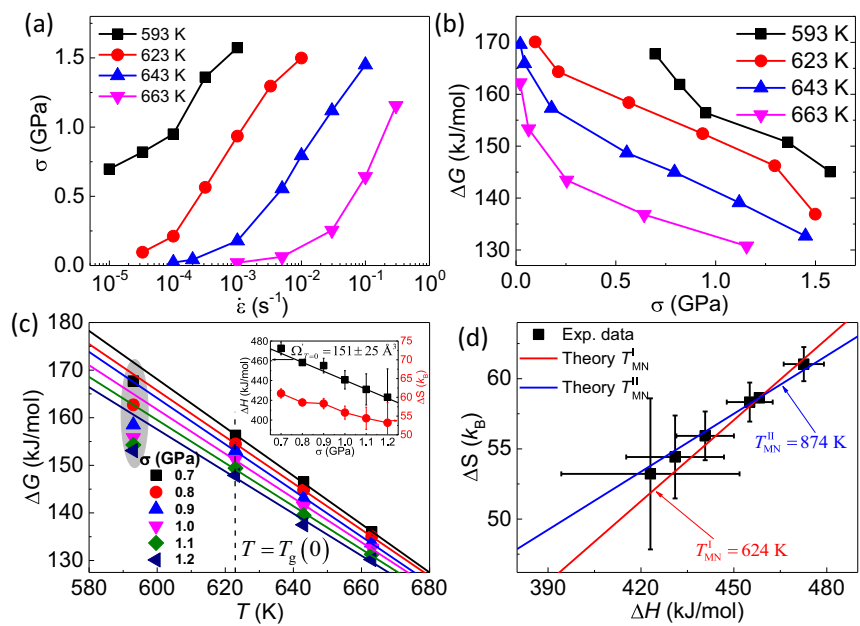

FIG. 4. (Color online) Experimental support of compensation rule via compression of Vit 1; the data are from Ref. [54]. (a) Strain-rate-modulated stress at different temperatures. (b) Activation free energy as a function of stress at different temperatures. (c) Linear fits of activation energy [above $T_{\mathrm{g}}(0)=624 \mathrm{~K}$ ] as a function of temperature. Data in the shaded area are excluded due to change of mechanism. Inset shows that the activation volume is in agreement with regime II. (d) Enthalpy-entropy compensation. The red line represents a best fit with $T_{\mathrm{MN}}^{\mathrm{I}}=T_{\mathrm{g}}(0)=624 \mathrm{~K}$; the blue line denotes a best fit with $T_{\mathrm{MN}}^{\mathrm{II}}=1.4 T_{\mathrm{g}}(0)=874 \mathrm{~K}$. The theoretical predictions qualitatively agree with experimental data, while $T_{\mathrm{MN}}^{\mathrm{II}}$ yields a better agreement. 
using $\Delta G(\sigma, T)=k_{B} T \ln \left(\dot{\varepsilon}_{0} / \dot{\varepsilon}\right)$, where $\dot{\varepsilon}$ is the compressive strain rate. Here we take the reference strain rate $\dot{\varepsilon}_{0}$ as half of $\dot{\gamma}_{0}$ because of a geometrical consideration [22,24,34]. $\Delta G$ is plotted against the stress at different temperatures in Fig. 4(b). For a first approximation, we extract $\Delta G$ as a function of temperature by linear interpolation of $\Delta G$ versus $\sigma$ data. The collected $\Delta G$ data are further shown versus temperature at different stress levels in Fig. 4(c). Linear fits of $\Delta G$ versus $T$ yield the $\Delta S$ and $\Delta H$ values. The data in the shaded area below $T_{\mathrm{g}}(0)=624 \mathrm{~K}$ are excluded because the mechanism changes near $T_{\mathrm{g}}(0)$, so that all of the experimental data are within regime II, as shown in Fig. 2(e). This is supported by the activation volume shown in the inset of Fig. 4(c). The derived activation volume of the order $151 \pm 25 \AA^{3}$ is in nice agreement with theoretical prediction of the order of $100 \AA^{3}$ in regime II; see Fig. 2(b).

Figure 4(d) plots all of the summarized data for the activation entropy versus the activation enthalpy of Vit 1 [54]. A linear relationship seems to be established between $\Delta S$ and $\Delta H$. To verify the findings in simulations, we provide two linear plots with a best fit of the compensation rule using compensation temperature $T_{\mathrm{MN}}^{\mathrm{I}}=T_{\mathrm{g}}(0)=624 \mathrm{~K}$ for regime $\mathrm{I}$ and $T_{\mathrm{MN}}^{\mathrm{II}}=1.4 T_{\mathrm{g}}(0)=874 \mathrm{~K}$ for regime $\mathrm{II}$, respectively. While both of the theoretical predictions are within the error range, $T_{\mathrm{MN}}^{\mathrm{II}}$ is in a better agreement with the experiment than $T_{\mathrm{MN}}^{\mathrm{I}}$. The experimental data are basically located in regime II considering the relatively high stress level $(\sigma / E=0 \sim 0.016$, where $E=96 \mathrm{GPa}$ is the Young's modulus of Vit 1 [54]) and the temperature range is above $T_{\mathrm{g}}(0)$. The quantity of activation volume shown in the inset of Fig. 4(c) also supports the point that these experimental data are within the supercooled liquid region.

The above analysis confirms the compensation rule using both atomistic simulations and experiments. The very high time resolution of MD allows us to study thermally activated processes happening during even an ephemeral homogenous deformation of an MG, which cannot be captured in experiments. However, MD can be used only for very short times and fast events. On the other hand, experiments enable the study of long time periods and slow events. However, the heterogeneous strain-rate distribution of localized shear deformation following homogenous deformation hinders a purely thermodynamic understanding of the intrinsic deformation unit of MGs based only on experiments. Hence, a study combining MD simulations and experiments provides an enthalpy-entropy compensation effect over a wide strain-rate regime. We also note that strain rate can sometimes change the deformation mechanisms of both crystals [53,55-57] and amorphous solids [58], which hinders a direct interpretation of experimental phenomena by atomistic simulations due to the time-scale limitation of the latter. The present study compares the high-strain-rate simulations with high-temperature experimental results, which is meaningful in terms of intrinsic homogenous flow behavior of MGs which are accommodated by STZs or other diffusive mechanisms.

\section{DISCUSSION}

Before we discuss any physical meaning of the compensation rule and compensation temperatures in $\mathrm{MG}$, we carry out

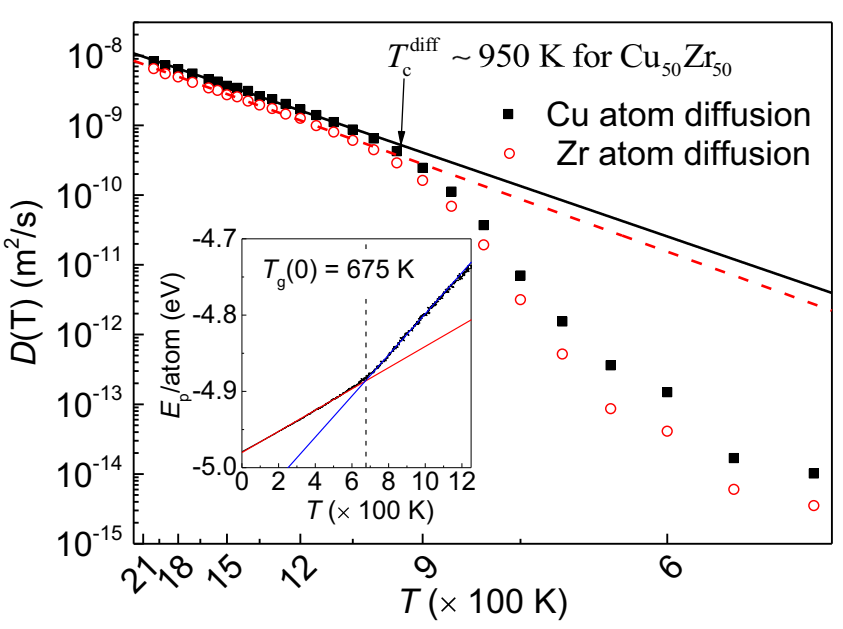

FIG. 5. (Color online) Understanding of critical temperatures of MGs, which correlate with $T_{\mathrm{MN}}$. Arrhenius plot of the diffusion coefficient against temperature in the $\mathrm{Cu}_{50} \mathrm{Zr}_{50}$. A critical temperature $T_{\mathrm{c}}^{\text {diff }} \sim 950 \mathrm{~K} \sim 1.4 T_{\mathrm{g}}(0)$ exists at which diffusion deviates from the Arrhenius relationship from the high-temperature limit. Atomic diffusion starts to feel the roughness of the free-energy surface. This critical temperature agrees with $T_{\mathrm{MN}}^{\mathrm{II}}=970 \pm 40 \mathrm{~K}$ for regime II. Inset shows the cooling history, which yields $T_{\mathrm{g}}(0)=675 \mathrm{~K}$, coinciding with $T_{\mathrm{MN}}^{\mathrm{I}}=687 \pm 51 \mathrm{~K}$ for regime $\mathrm{I}$.

statistical analysis which indicates a physical cause behind the identified compensation effect in MGs; see details in Fig. S4 of the Supplemental Material [51]. We may understand the compensation rule in terms of basic thermodynamics. By combining Eqs. (2) and (5), the activation enthalpy can be expressed as $\Delta H=T \Delta S+k_{B} T \ln \left(\dot{\gamma}_{0} / \dot{\gamma}\right)$. When the temperature approaches $T_{\mathrm{MN}}$, the strain rate $\dot{\gamma}$ will reach its upper limit, i.e., $\dot{\gamma}_{0}$. If the activation entropy is assumed to be insensitive to temperature [as shown in Fig. S3 of the Supplemental Material [51] and Fig. 4(c)], the above expression yields the original form of the $\mathrm{MN}$ rule, i.e., $\Delta S=\Delta H / T_{\mathrm{MN}}$. The physical meaning seems to be that $\Delta G$ of a specific deformation mechanism tends to be a negligible value when the temperature approaches $T_{\mathrm{MN}}$ only if the deformation mechanism is dominant and holds up to $T_{\mathrm{MN}}$. However, the deformation mechanism may already shift before reaching $T_{\mathrm{MN}}$. Therefore, $T_{\mathrm{MN}}$ can be a theoretical upper limit that specifies a unique deformation mechanism in a specific regime of the deformation map.

To further understand the physical meaning, we analyze the temperature-dependent diffusion kinetics. Figure 5 shows the Arrhenius plot of both $\mathrm{Cu}$ and $\mathrm{Zr}$ atoms against the reciprocal temperature. At the high-temperature limit, diffusion obeys an Arrhenius relation in the liquid region. At a critical temperature $T_{\mathrm{c}}^{\text {diff }} \sim 950 \mathrm{~K}$, a kink appears in the Arrhenius plot. Diffusion starts to deviate from the linear relationship once it feels the roughness of the free-energy surface, while the inset of Fig. 5 shows the cooling history, which yields $T_{\mathrm{g}}(0)=675 \mathrm{~K}$.

Therefore, $T_{\mathrm{MN}}^{\mathrm{I}}$ is in agreement with the thermal glass transition temperature. This is not surprising since the glass transition occurs at $T_{\mathrm{g}}(0)$, where structural changes occur and the deformation mechanism shifts. $T_{\mathrm{MN}}^{\mathrm{II}}=970 \pm 40 \mathrm{~K}$ matches the critical temperature $T_{\mathrm{c}}^{\text {diff }} \sim 950 \mathrm{~K} \sim 1.4 T_{\mathrm{g}}(0)$, 
where diffusion deviates from the conventional Arrhenius relation. The deviation indicates that diffusion kinetics start to feel the roughness of the free-energy landscape, which also suggests a deformation mechanism shift at this critical temperature. In this sense, $1.4 T_{\mathrm{g}}(0)$ is the critical temperature, above which the supercooled liquid loses the memory of previous thermal and mechanical histories [59]. Therefore, $T_{\mathrm{MN}}^{\mathrm{II}}$ could be a unique theoretical temperature indicative of the deformation mechanism in the supercooled liquid state (regime II). For comparison, we also conduct mode-coupling theory (MCT) analyses in Fig. S5 of the Supplemental Material [51,60,61]. The MCT critical temperature is $T_{\mathrm{c}} \sim 780 \mathrm{~K}$ for $\mathrm{Cu}_{50} \mathrm{Zr}_{50}$, which is well below the estimated $T_{\mathrm{MN}}^{\mathrm{II}}$ but above $T_{\mathrm{MN}}^{\mathrm{I}} \cdot$

\section{CONCLUSIONS}

To summarize, atomistic simulations and experiments uncover a universal enthalpy-entropy compensation rule in MGs. It stands in a wide temperature and stress range spanning the glass transition induced by temperature and/or stress. Distinct compensation temperatures are discovered for each regime of the deformation map. Before the glass transition, $T_{\mathrm{MN}}$ is correlated with $T_{\mathrm{g}}(0)$, which could be a theoretical upper temperature limit of the STZ. On the other hand, $T_{\mathrm{MN}} \sim 1.4 T_{\mathrm{g}}(0)$ for supercooled liquid is possibly the theoretical upper temperature limit of the $\alpha$ relaxation, where the diffusion deviates from the Arrhenius relationship because the free-energy surface becomes rough.

\section{ACKNOWLEDGMENTS}

This work was financially supported by the NSFC (Grants No. 11132011, No. 11402269, and No. 11472287), the National Key Basic Research Program of China (Grant No. 2012CB937500), and the CAS/SAFEA International Partnership Program for Creative Research Teams. S.O. acknowledges support by KAKENHI Grants No. 23246025 [Scientific Research (A)], No. 22102003 (Scientific Research on Innovative Area), and No. 25630013 (Challenging Exploratory Research), and Elements Strategy Initiative for Structural Materials (ESISM).
[1] O. Exner, Concerning the isokinetic relationship, Nature (London) 201, 488 (1964).

[2] R. R. Krug, W. G. Hunter, and R. A. Grieger, Statistical interpretation of enthalpy-entropy compensation, Nature (London) 261, 566 (1976).

[3] W. Good, A biological example of the compensation law, Nature (London) 214, 1250 (1967).

[4] G. Boisvert, L. J. Lewis, and A. Yelon, Many-Body Nature of the Meyer-Neldel Compensation Law for Diffusion, Phys. Rev. Lett. 75, 469 (1995).

[5] W. Jiang, Q. Kong, D. Molodov, and G. Gottstein, Compensation effect in grain boundary internal friction, Acta Mater. 57, 3327 (2009).

[6] S. Hara and J. Li, Adaptive strain-boost hyperdynamics simulations of stress-driven atomic processes, Phys. Rev. B 82, 184114 (2010).

[7] S. Ryu, K. Kang, and W. Cai, Entropic effect on the rate of dislocation nucleation, Proc. Natl. Acad. Sci. USA 108, 5174 (2011).

[8] Y.-J. Wang, A. Ishii, and S. Ogata, Entropic effect on creep in nanocrystalline metals, Acta Mater. 61, 3866 (2013).

[9] W. Meyer and H. Neldel, Relation between the energy constant $\varepsilon$ and the quantity constant $\alpha$ in the conductivity-temperature formula of oxide semiconductors, Z. Tech. Phys. (Leipzig) 12, 588 (1937).

[10] A. Yelon, Microscopic Explanation of the Compensation (Meyer-Neldel) Rule, Phys. Rev. Lett. 65, 618 (1990).

[11] A. Yelon, B. Movaghar, and R. S. Crandall, Multi-excitation entropy: its role in thermodynamics and kinetics, Rep. Prog. Phys. 69, 1145 (2006).

[12] H. W. Sheng, W. K. Luo, F. M. Alamgir, J. M. Bai, and E. Ma, Atomic packing and short-to-medium-range order in metallic glasses, Nature (London) 439, 419 (2006).

[13] A. Hirata, L. J. Kang, T. Fujita, B. Klumov, K. Matsue, M. Kotani, A. R. Yavari, and M. W. Chen, Geometric frustration of icosahedron, Science 341, 376 (2013).
[14] E. Ma, Tuning order in disorder, Nat. Mater. 14, 547 (2015).

[15] H. Eyring, The activated complex in chemical reactions, J. Chem. Phys. 3, 107 (1935).

[16] T. T. Lau, A. Kushima, and S. Yip, Atomistic Simulation of Creep in a Nanocrystal, Phys. Rev. Lett. 104, 175501 (2010).

[17] M. Kabir, T. T. Lau, D. Rodney, S. Yip, and K. J. Van Vliet, Predicting Dislocation Climb and Creep from Explicit Atomistic Details, Phys. Rev. Lett. 105, 095501 (2010).

[18] D. Teschner, G. Novell-Leruth, R. Farra, A. Knop-Gericke, R. Schlögl, L. Szentmiklósi, M. González Hevia, H. Soerijanto, R. Schomäcker, J. Pérez-Ramírez, and N. López, In situ surface coverage analysis of $\mathrm{RuO}_{2}$-catalysed $\mathrm{HCl}$ oxidation reveals the entropic origin of compensation in heterogeneous catalysis, Nat. Chem. 4, 739 (2012).

[19] P. Koziatek, J.-L. Barrat, P. Derlet, and D. Rodney, Inverse Meyer-Neldel behavior for activated processes in model glasses, Phys. Rev. B 87, 224105 (2013).

[20] F. Faupel, D. Kiel, W. Frank, K. Ra, and H. R. Schober, Diffusion in metallic glasses and supercooled melts, Rev. Mod. Phys. 75, 237 (2003).

[21] Y. Cheng and E. Ma, Atomic-level structure and structureproperty relationship in metallic glasses, Prog. Mater. Sci. 56, 379 (2010).

[22] A. S. Argon, Plastic deformation in metallic glasses, Acta Metallurg. 27, 47 (1979).

[23] M. L. Falk and J. S. Langer, Dynamics of viscoplastic deformation in amorphous solids, Phys. Rev. E 57, 7192 (1998).

[24] W. L. Johnson and K. Samwer, A Universal Criterion for Plastic Yielding of Metallic Glasses with a $\left(T / T_{g}\right)^{2 / 3}$ Temperature Dependence, Phys. Rev. Lett. 95, 195501 (2005).

[25] W. L. Johnson, M. D. Demetriou, J. S. Harmon, M. L. Lind, and K. Samwer, Rheology and properties of metallic glass-forming liquids: A potential energy landscape perspective, MRS Bull. 32, 644 (2007). 
[26] F. Spaepen, A microscopic mechanism for steady state inhomogeneous flow in metallic glasses, Acta Metallurg. 25, 407 (1977).

[27] Z. Lu, W. Jiao, W. H. Wang, and H. Y. Bai, Flow Unit Perspective on Room Temperature Homogeneous Plastic Deformation in Metallic Glasses, Phys. Rev. Lett. 113, 045501 (2014).

[28] Z. Wang, B. A. Sun, H. Y. Bai, and W. H. Wang, Evolution of hidden localized flow during glass-to-liquid transition in metallic glass, Nat. Commun. 5, 5823 (2014).

[29] S. V. Ketov, Y. H. Sun, S. Nachum, Z. Lu, A. Checchi, A. R. Beraldin, H. Y. Bai, W. H. Wang, D. V. Louzguine-Luzgin, M. A. Carpenter, and A. L. Greer, Rejuvenation of metallic glasses by non-affine thermal strain, Nature (London) 524, 200 (2015).

[30] M. Q. Jiang, Z. Ling, J. X. Meng, and L. H. Dai, Energy dissipation in fracture of bulk metallic glasses via inherent competition between local softening and quasi-cleavage, Philos. Mag. 88, 407 (2008).

[31] X. Huang, Z. Ling, and L. H. Dai, Ductile-to-brittle transition in spallation of metallic glasses, J. Appl. Phys. 116, 143503 (2014).

[32] Y. Fan, T. Iwashita, and T. Egami, How thermally activated deformation starts in metallic glass, Nat. Commun. 5, 5083 (2014).

[33] Y. Fan, T. Iwashita, and T. Egami, Crossover from Localized to Cascade Relaxations in Metallic Glasses, Phys. Rev. Lett. 115, 045501 (2015).

[34] C. Schuh, T. Hufnagel, and U. Ramamurty, Mechanical behavior of amorphous alloys, Acta Mater. 55, 4067 (2007).

[35] D. Pan, A. Inoue, T. Sakurai, and M. W. Chen, Experimental characterization of shear transformation zones for plastic flow of bulk metallic glasses, Proc. Natl. Acad. Sci. USA 105, 14769 (2008).

[36] Y. H. Liu, T. Fujita, D. P. B. Aji, M. Matsuura, and M. W. Chen, Structural origins of Johari-Goldstein relaxation in a metallic glass, Nat. Commun. 5, 3238 (2014).

[37] S. Plimpton, Fast parallel algorithms for short-range molecular dynamics, J. Comput. Phys. 117, 1 (1995).

[38] Y. Q. Cheng, E. Ma, and H. W. Sheng, Atomic Level Structure in Multicomponent Bulk Metallic Glass, Phys. Rev. Lett. 102, 245501 (2009).

[39] P. Guan, M. Chen, and T. Egami, Stress-Temperature Scaling for Steady-State Flow in Metallic Glasses, Phys. Rev. Lett. 104, 205701 (2010).

[40] P. Guan, S. Lu, M. J. B. Spector, P. K. Valavala, and M. L. Falk, Cavitation in Amorphous Solids, Phys. Rev. Lett. 110, 185502 (2013).

[41] Y. Cheng and E. Ma, Intrinsic shear strength of metallic glass, Acta Mater. 59, 1800 (2011).

[42] J. Das, M. B. Tang, K. B. Kim, R. Theissmann, F. Baier, W. H. Wang, and J. Eckert, "Work-Hardenable" Ductile Bulk Metallic Glass, Phys. Rev. Lett. 94, 205501 (2005).

[43] W. H. Wang, The elastic properties, elastic models and elastic perspectives of metallic glasses, Prog. Mater. Sci. 57, 487 (2012).
[44] A. Greer, Y. Cheng, and E. Ma, Shear bands in metallic glasses, Mater. Sci. Eng. Rep. 74, 71 (2013).

[45] M. Parrinello, Polymorphic transitions in single crystals: A new molecular dynamics method, J. Appl. Phys. 52, 7182 (1981).

[46] D. Rodney and C. Schuh, Distribution of Thermally Activated Plastic Events in a Flowing Glass, Phys. Rev. Lett. 102, 235503 (2009).

[47] F. Shimizu, S. Ogata, and J. Li, Theory of shear banding in metallic glasses and molecular dynamics calculations, Mater. Trans. 48, 2923 (2007).

[48] J. Li, AtomEye: an efficient atomistic configuration viewer, Model. Simul. Mater. Sci. Eng. 11, 173 (2003).

[49] U. F. Kocks, A. S. Argon, and M. F. Ashby, Thermodynamics and kinetics of slip, Prog. Mater. Sci. 19, 1 (1975).

[50] L. Li, E. Homer, and C. Schuh, Shear transformation zone dynamics model for metallic glasses incorporating free volume as a state variable, Acta Mater. 61, 3347 (2013).

[51] See Supplemental Material at http://link.aps.org/supplemental/ 10.1103/PhysRevB.92.174118 for more details of simulations, more evidence and discussion on the glass transition, and statistical and mode-coupling theory analysis of the enthalpyentropy compensation rule in MGs.

[52] A. J. Liu and S. R. Nagel, Nonlinear dynamics: Jamming is not just cool any more, Nature (London) 396, 21 (1998).

[53] T. Zhu, J. Li, A. Samanta, A. Leach, and K. Gall, Temperature and Strain-Rate Dependence of Surface Dislocation Nucleation, Phys. Rev. Lett. 100, 025502 (2008).

[54] J. Lu, G. Ravichandran, and W. Johnson, Deformation behavior of the $\mathrm{Zr}_{41.2} \mathrm{Ti}_{13.8} \mathrm{Cu}_{12.5} \mathrm{Ni}_{10} \mathrm{Be}_{22.5}$ bulk metallic glass over a wide range of strain-rates and temperatures, Acta Mater. 51, 3429 (2003).

[55] Y. Fan, Y. N. Osetsky, S. Yip, and B. Yildiz, Onset Mechanism of Strain-Rate-Induced Flow Stress Upturn, Phys. Rev. Lett. 109, 135503 (2012).

[56] C. R. Weinberger, A. T. Jennings, K. Kang, and J. R. Greer, Atomistic simulations and continuum modeling of dislocation nucleation and strength in gold nanowires, J. Mech. Phys. Solids 60, 84 (2012).

[57] Y. Fan, Y. N. Osetskiy, S. Yip, and B. Yildiz, Mapping strain rate dependence of dislocation-defect interactions by atomistic simulations, Proc. Natl. Acad. Sci. USA 110, 17756 (2013).

[58] D. Rodney, A. Tanguy, and D. Vandembroucq, Modeling the mechanics of amorphous solids at different length scale and time scale, Model. Simul. Mater. Sci. Eng. 19, 083001 (2011).

[59] M. Wakeda, J. Saida, J. Li, and S. Ogata, Controlled rejuvenation of amorphous metals with thermal processing, Sci. Rep. 5, 10545 (2015).

[60] J. M. Dickey and A. Paskin, Computer simulation of the lattice dynamics of solids, Phys. Rev. 188, 1407 (1969).

[61] W. Gotze and L. Sjogren, Relaxation processes in supercooled liquids, Rep. Prog. Phys. 55, 241 (1992). 